\title{
How can mental health and substance use services become dual diagnosis capable? Moving from theory into practice
}

\author{
Petra Staiger ${ }^{1 *}$, Alexandra Howard ${ }^{1,2}$, Anna C Thomas ${ }^{1,2}$, Greg Young ${ }^{3}$, Marita McCabe ${ }^{1}$ \\ From Health Services Research: Evidence-based practice \\ London, UK. 1-3 July 2014
}

\section{Background}

There is evidence that separate service delivery for individuals with dual Mental Health and Substance Use Disorders is expensive and can have negative treatment implications. While redesigning the health system to provide integrative care is optimal, costs of this mean that the focus has generally been on developing models of care and best practice guidelines to improve the identification and treatment of dual diagnosis within current systems. 'Shared care' with $\mathrm{MH}$ and SUD services collaborating to coordinate delivery of interventions is considered the best option within these parameters. However, only a few projects have reported on the implementation of such highly collaborative models and many of these have not yet been systematically evaluated. The current study represents the implementation and evaluation of an Early Identification Model (EIM) in three health services settings. The EIM was created to support health services through the complex dynamics of becoming dual diagnosis capable. It consists of two primary components: two-step systematic screening, and clearly defined care pathways to ensure that dual diagnosis identification leads to appropriate follow up, referral, or delivery of evidence-based interventions for each individual.

\section{Methods}

The model was implemented at three health service sites (mental health; alcohol and drug; community health). The methodology consisted of three components. First, the literature on dual diagnosis and health service systems was consulted; second, the findings of the evaluations (i.e., focus group data and quantitative findings) were reviewed from the three sites; and, finally, feedback from an expert

${ }^{1}$ School of Psychology, Deakin University, Burwood, Victoria, Australia Full list of author information is available at the end of the article advisory group who consisted of senior academics and clinicians in the field was incorporated.

\section{Results}

The four principles arrived at were to: (a) embed the model into existing systems; (b) establish clear guidelines for clinical decisionmaking and care pathways; (c) use a change management framework during the implementation phase; and (d) provide training and capacity building to staff.

\section{Conclusions}

Although the three services were able to successfully implement the new screening and referral pathway procedures with their available resources, it was clear that additional funding would be required in the longer term to accommodate more integrated in-house treatment of clients with a dual diagnosis. SUD staff in particular felt they did not have the required level of skills to treat $\mathrm{MH}$ issues so further training in mental health management would be useful in the longer term. Investment in training staff is likely to substantially improve client outcomes without the need for immediate structural change in the current health care system.

\section{Authors' details}

${ }^{1}$ School of Psychology, Deakin University, Burwood, Victoria, Australia. ${ }^{2}$ Australian Institute of Family Studies, Victoria, Australia. ${ }^{3}$ Southern Health Care Network, Clayton, Victoria, Australia.

Published: 7 July 2014

doi:10.1186/1472-6963-14-S2-P116

Cite this article as: Staiger et al:: How can mental health and substance use services become dual diagnosis capable? Moving from theory into practice. BMC Health Services Research 2014 14(Suppl 2):P116. 